\title{
The 3Rs in Animal Welfare Bodies at Swedish Universities - Knowledge, Attitudes, Implementation
}

\author{
Johan Lindsjö1,2, Charlotte Berg 3 , Ulf Olsson ${ }^{4}$ and Elin Törnqvist ${ }^{5}$ \\ ${ }^{1}$ Department of Animal Environment and Health, Swedish University of Agricultural Sciences, Uppsala, Sweden; ${ }^{2}$ Swedish Centre for Animal Welfare, \\ Swedish University of Agricultural Sciences, Uppsala, Sweden; ${ }^{3}$ Department of Animal Environment and Health, Swedish University of Agricultural \\ Sciences, Skara, Sweden; ${ }^{4}$ Unit of Applied Statistics and Mathematics, Swedish University of Agricultural Sciences, Uppsala, Sweden; ${ }^{5}$ Institute of \\ Environmental Medicine, Karolinska Institutet, Solna, Sweden
}

\begin{abstract}
The implementation of the 3Rs (replacement, reduction and refinement) is emphasized in EU Directive 2010/63. The task of the animal welfare bodies (AWB) is to strengthen animal welfare and develop the 3 Rs at research animal facilities. In 2016, we surveyed the knowledge on, attitudes towards and implementation of the 3 Rs within AWBs at eight major Swedish universities. Based on responses of 34 closed-ended questions from 44 of 90 AWB members, the overall attitude towards the 3 Rs was positive. AWB members did not believe that the 3 Rs slow down innovation or result in increased costs, and refinement was considered beneficial for research quality. AWB members were particularly positive towards refinement questions in the survey. A majority of the AWB members predicted that alternative methods will never replace animal use. Researchers as a group represented in the AWBs were significantly less positive towards the $3 R s$ compared to the group of veterinarians. The tasks of the AWBs, e.g., giving advice on the 3 Rs and following up on animal use in projects, were often not carried out in the AWB or not known by the respondents. Our results indicate a need for more practical and regulatory guidance and support to the AWBs. To reach the goal of the EU Directive to phase out animal use in research and education, we suggest that technical expertise in replacement techniques is included in the AWBs. We emphasize the need to strengthen the awareness of the 3 Rs among researchers at Swedish universities.
\end{abstract}

\section{Introduction}

The implementation of the 3Rs (replace, reduce, refine) in practice is emphasized in European Union (EU) Directive 2010/63 on the protection of animals used for scientific purposes, which states the clear intention to strive towards replacement of animal use in research (EU, 2010). Directive 2010/63 was implemented into Swedish legislation in 2013, and, consequently, setting up local animal welfare bodies (AWBs) is mandatory for breeders, suppliers and users of research animals. According to the Directive, an AWB within a research animal facility "shall, as a minimum, carry out the following tasks: (a) advise the staff dealing with animals on matters related to the welfare of animals, in relation to their acquisition, accommodation, care and use; (b) advise the staff on the application of the re- quirement of replacement, reduction and refinement, and keep it informed of technical and scientific developments concerning the application of that requirement; (c) establish and review internal operational processes as regards monitoring, reporting and follow-up in relation to the welfare of animals housed or used in the establishment; (d) follow the development and outcome of projects, taking into account the effect on the animals used, and identify and advise as regards elements that further contribute to replacement, reduction and refinement; and (e) advise on rehoming schemes, including the appropriate socialisation of the animals to be rehomed". The animal welfare body "shall include at least the person or persons responsible for the welfare and care of the animals, and in the case of a user, a scientific member", and also receive input from a designated veterinarian or another suitably qualified expert (EU, 2010).
Received November 14, 2019: Accepted March 11, 2021

Epub March 29, 2021; ( The Authors, 2021.

ALTEX 38(3), 477-489. doi:10.14573/altex.1911141

Correspondence: Johan Lindsjö, DVM, M.Sc.

Department of Animal Environment and Health

Swedish University of Agricultural Sciences

Ulls väg 26, 75651 Uppsala, Sweden

(johan.lindsjo@slu.se)
This is an Open Access article distributed under the terms of the Creative Commons Attribution 4.0 International license (http://creativecommons.org/licenses/by/4.0/) which permits unrestricted use, distribution and reproduction in any medium, provided the original work is appropriately cited. 
In a review by the European Commission regarding the implementation of Directive 2010/63 into national legislation, users and other stakeholders considered the AWBs a positive and effective requirement that is contributing to increased awareness of the 3Rs, improving animal use, and promoting a culture of care. However, in some member states, the role of the AWBs is unclear in relation to the primary evaluation of projects, and some AWBs have not developed suitable information strategies for alternatives (EU, 2017).

The 3Rs were originally developed to replace the use of animals in scientific procedures, limit the number of animals used, and improve laboratory animal welfare (Russell and Burch, 1959; Executive Committee of the Congress, 2009). Over the years, the 3Rs have been adapted to research in different areas, such as biomedicine, toxicology, agriculture and wildlife/ecosystems to guide scientists on the ethical use of animals in research, testing, and education (Norecopa, 2012; Hubrecht, 2014; Lindsjö et al., 2016). During 2016, a total of 350,664 research animals (according to the definition in the EU Directive 2010/63) were used in Sweden; 89\% of these animals, i.e., traditional laboratory, farm and wild animals comprising different research areas and sometimes education, were used at the eight Swedish universities included in this study (Ljung and Bornestaf, 2018).

To raise awareness, recognize possibilities, identify obstacles, and thus facilitate the implementation of the $3 \mathrm{Rs}$, it is important to understand how the 3 Rs are perceived among stakeholders in academia, regulatory bodies and industry. Nøhr et al. (2016) found in a Danish survey that the interest in the 3Rs of researchers in the private sector was generally higher compared to that of researchers in the public sector. British, Dutch and Danish surveys have shown that researchers involved in animal experiments, animal care staff and animal welfare officers have an overall good knowledge and a positive attitude towards the 3Rs (NC3Rs, 2008; Leenars et al., 2009; van Luijk et al., 2011; Nøhr et al., 2016). In contrast, the overall attitude was found to be less favorable in a Canadian survey of researchers (Fenwick et al., 2011), and Knight et al. (2009) suggested that researchers working with animals might be more prone to accept the use of animals in experiments than other societal groups.

The incorporation of the $3 \mathrm{Rs}$ in animal ethics committees (AECs) is an important part of the review of animal-use protocols. In a Canadian survey with AEC members, Schuppli and Fraser (2005) found a lack of awareness of the 3Rs that could impede complete application of the 3 Rs. In contrast, Houde et al. (2009) found that the knowledge of the 3Rs was good in a smaller survey of a Canadian Institutional Animal Care and Use Committee (IACUC). Researchers and other stakeholders found that the availability, validation and implementation of the $3 \mathrm{R}$ strategies differ. In general, replacement is seen as the most difficult strategy to implement or give advice on, whereas reduction and refinement are perceived as easier to apply (Schiffelers et al., 2007; NC3Rs, 2008; van Luijk et al., 2013; Franco et al., 2018) and thus are more accepted strategies (Nøhr et al., 2016).
The aims of this study were to survey the knowledge of and the attitude towards the 3Rs among members of AWBs at the eight Swedish universities at which animals are used for scientific purposes and to identify similarities and differences between professional groups represented in the AWBs. We based our survey on the following research questions:

- Do the AWBs implement Directive 2010/63?

- Are there differences in attitude towards replacement, reduction and refinement among the AWB members?

- Are there differences between professional groups represented in the AWBs?

We hypothesized that the awareness and implementation of the 3Rs and the EU Directive 2010/63 differ between replacement, reduction and refinement, and between the professional groups represented in the AWBs. The results of the study identify challenges and opportunities for continued implementation of the 3Rs at Swedish universities, with bearing also on other countries, especially within the EU.

\section{Materials and methods}

All eight Swedish universities at which animals are used in research or education were identified and included in this study. A survey consisting of 34 questions was sent by e-mail to the chairpersons of the AWBs for distribution to all 90 representatives of the 10 AWBs during 2016 (Appendix $1^{1}$ ). Question items were built on previous surveys done by the $3 \mathrm{R}$ centers in the UK (NC3Rs, 2008) and Denmark (Nøhr et al., 2016) (QI). Some questions were identical or similar, but the answer alternatives were not always consistent between the surveys, e.g., scaled answers vs. direct proportion. Furthermore, the items did not include all, or completely identical, answer alternatives. This however still enabled comparison with the data on the knowledge and attitude towards the 3 Rs of British researchers and animal care staff and of Danish researchers.

The definitions of the 3Rs were originally proposed by Russell and Burch (1959), and similar definitions were used by NC3Rs (2008) and Nøhr et al. (2016). Possible misconceptions of the 3R definitions were also included by NC3Rs (2008) and Nøhr et al. (2016). We added questions about refinement to identify differences in attitude and implementation of the different Rs (QII). We also added questions about education, because animals used in education are included in the definition of research animals (QII). Finally, two sections were included to further understand the implementation of Directive 2010/63 in the AWBs (QIII) and what key factors may increase the implementation of the 3 Rs at the universities (QIV).

Before distribution, the survey was tested and commented on by three persons with research animal background. The survey, available in a Swedish and an English version, consisted of 29 quantitative questions with multiple choice, single checkbox or scaled answers ( 0 , don't know; 1 , strongly disagree; 2 ,

1 doi:10.14573/altex.1911141s 
disagree; 3, neither disagree/agree; 4, agree; 5, strongly agree). The survey focused on four areas: I) understanding of and attitudes to the 3Rs (QI.1-5), II) implementation of the 3Rs (QII. 1-10), III) implementation of EU Directive 2010/63 (QIII.1-8), and IV) key factors for successful implementation of the 3Rs at the university (QIV.1-6). The questions were closed-ended with possibilities to leave comments for some of the answers. The survey also included five demographic questions.

We defined the following six professional groups in the AWBs: persons responsible for animal welfare; researchers; veterinarians or other experts (termed veterinarians in this manuscript); animal technicians; ethologists; a group of miscellaneous professions. Despite a rather high response rate, the total number of respondents was too low to enable comparisons between universities or between age groups. We therefore only compared among the different Rs and among the professional groups represented in the AWBs. The results of the survey were evaluated using descriptive analysis (proportions, mean values). The analysis was based on answers from 44 respondents (41 in Swedish and three in English), except for questions where mean values were calculated, in which case the respondents who answered "don't know" were excluded.

In order to analyze differences between the professional groups in the AWBs (veterinarians, animal technicians, researchers, persons responsible for animal welfare, ethologists and others), two statistical analyses were performed; ordinal logistic methods and factor analyses. Calculations included all professional groups, but results from the group of ethologists (only one participant in the study) and from the group of miscellaneous professions (only four participants in the study) were excluded. Most of the questions were of Likert type with 5 categories, from 1 (strongly disagree) to 5 (strongly agree). Thus, the measurement scale is ordinal. Therefore, we analyzed them using ordinal logistic methods (see e.g., Olsson, 2002); all variables with numeric responses were analyzed.

The Glimmix procedure of the SAS (2017) package was used. The models were specified using a multinomial distribution and a cumulative logit link function. All questions of relevant type were analyzed using a custom-written SAS macro, and questions with non-numeric variables were excluded. This resulted in 140 analyses. Statistical significance was assigned when $p<0.05$. Since so many analyses were made, there was a considerable risk of mass significance. This means that out of 100 statistical tests we could expect 5 to show significance at the 5\% level, even if no real differences exist. Still, we have chosen to present the significant results, but we ask the reader to keep the risk of mass significance in mind. In an attempt to summarize the large number of variables into a smaller number of factors, a few factor analyses (see e.g., Morrison, 1976) were made. Since the variables are ordinal, the analyses were performed using polychoric correlations (Olsson, 1979) calculated using the Corr procedure in SAS (2017). Thereafter, the factor procedure of the SAS (2017) package was used for the factor analyses. The factors were Varimax-rotated to facilitate interpretation. Questions with a 5-graded response rating from strongly disagree to strongly agree (QI.4-5, QII.3-5, QIII.4-5, QIV.4-5) were included in the factor analyses.
Before performing the analyses, all variables were categorized as replacement-, reduction- and refinement-related responses or as a more general response including all 3Rs. In addition, all questions were divided into positive and negative attitude to the 3Rs. In total, 75 questions were analyzed. The majority of the questions were refinement-related (38); the rest were distributed between replacement (13), reduction (11) and general 3R (13). Fifteen questions were categorized as negative effects of the $3 \mathrm{Rs}$, 54 questions as positive effects, and six questions were regarded as neutral before analyses (Appendix $2^{1}$ ).

For each factor, differences between professional groups in the AWBs were tested using ANOVA. Follow-up analyses, i.e., pairwise comparisons, were adjusted for multiplicity using Tukey-Kramer's method. Statistical significance was assigned when $\mathrm{p}<0.05$. The assumptions were checked by preparing diagnostic plots; no apparent deviations from normality or homoscedasticity were detected.

The participants' privacy and anonymity were fully protected in this project.

\section{Results}

In total, 44 members of the AWBs, i.e., $49 \%$, responded to the survey. The respondents included 14 persons responsible for animal welfare, nine researchers, ten veterinarians or other experts ("veterinarians"), six animal technicians, one ethologist and four persons in the group of miscellaneous professions. The size of the AWBs differed between the universities, with a range from 3 to 14 AWB members. All eight universities were represented among the respondents, although the response rate varied between the AWBs from $10 \%$ to $85 \%$. The median age span among the respondents was 50-59 (from 25 to over 65 years), and both genders were represented ( $41 \%$ men and 59\% women). Remarkably, for several questions, the proportion of the answer "I don't know" was high, e.g., regarding the implementation of the EU Directive 2010/63, up to $43 \%$.

\subsection{Knowledge and attitudes towards the 3Rs}

With some exceptions, the definitions of the 3Rs (Question [Q] I.1-3) were well understood. 93\% of the respondents correctly recognized in vitro/in silico methods as replacement. Replacing more sentient mammals with less sentient mammals is not replacement, which was correctly recognized by $86 \%$. However, only $27 \%$ of the respondents answered that replacement includes replacing vertebrates with invertebrates, indicating a knowledge gap regarding the definition of replacement according to the EU Directive (Fig. 1A). The concept of reduction was also well understood by the respondents. $98 \%$ answered that reduction is consistent with obtaining comparable levels of information while using fewer animals in an experiment, and 61\% recognized that obtaining more information from an experiment while using the same number of animals fits the definition of reduction. $20 \%$ incorrectly suggested that reducing pain and suffering caused by the research procedures is reduction (Fig. 1B). Improving experimental procedures to decrease the animals' experience of pain 


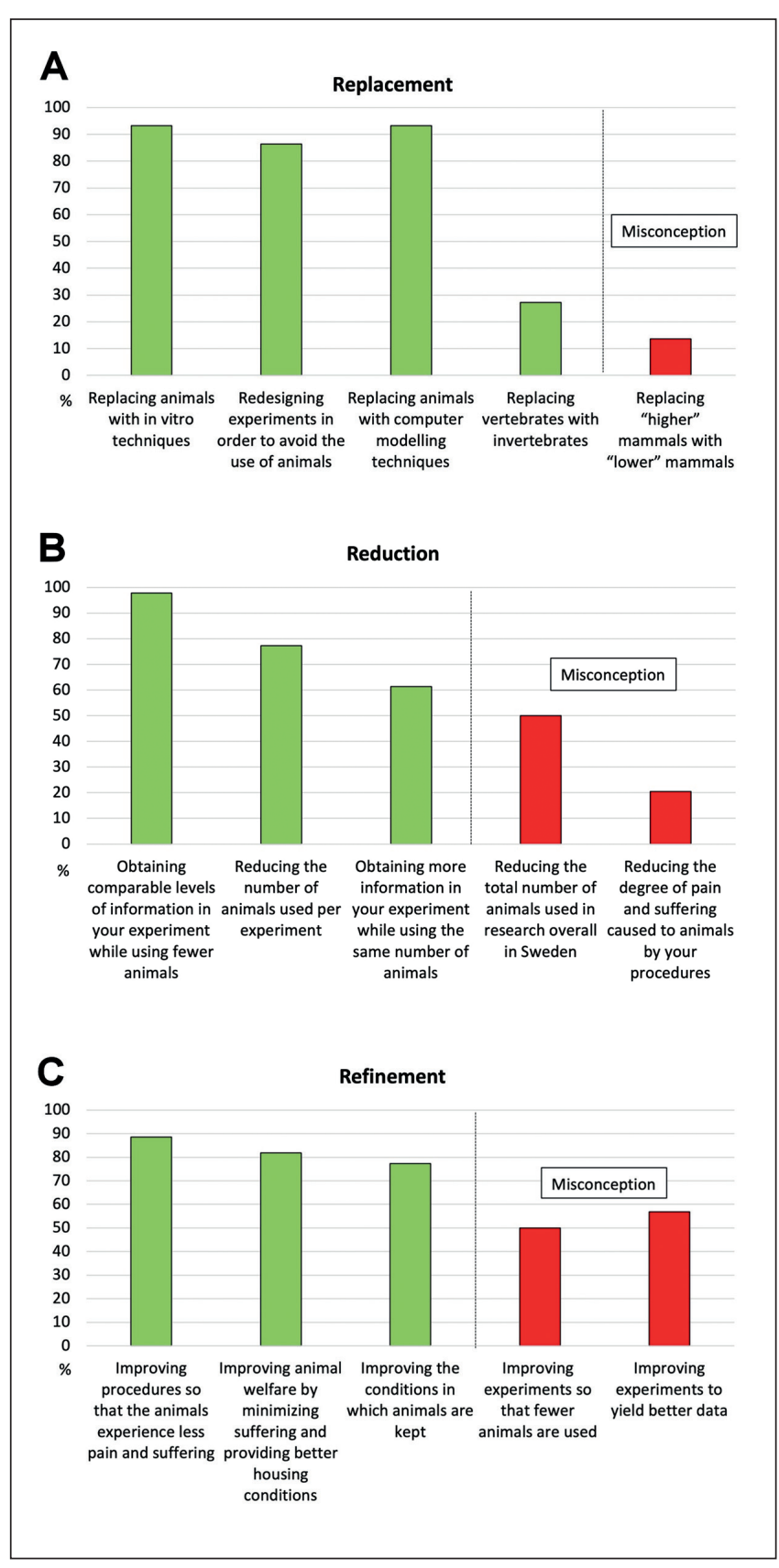

Fig. 1: AWB members' understanding of 3R definitions "Which of the following definitions fits your understanding of (A) Replacement, (B) Reduction, and (C) Refinement?" (QI.1-3). Responses (multiple choice, where several answers are appropriate) presented as percentage. $\mathrm{N}=44$.

and suffering as well as improving the conditions in which animals are kept were well recognized as refinement (89\% and $77 \%$ of the respondents, respectively). At least half of the respondents wrongly defined refinement as improving experiments so that fewer animals are used or improving experiments to yield better data (50 and 57\%, respectively) (Fig. 1C).
A

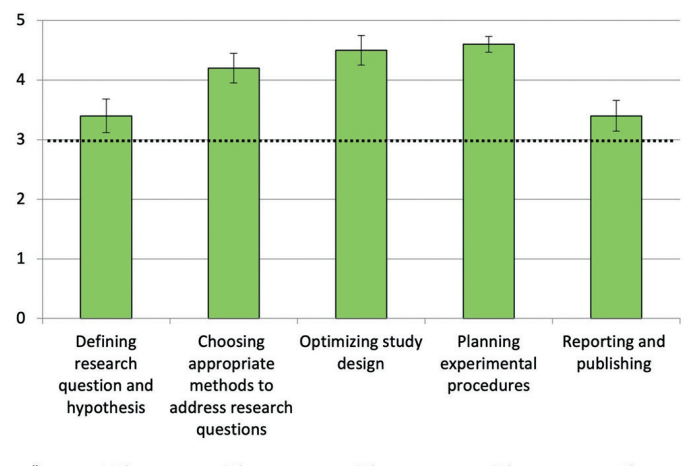

$\begin{array}{llllll}\text { "I don't know" } & 11 \% & 9 \% & 9 \% & 0 \% & 7 \%\end{array}$

B

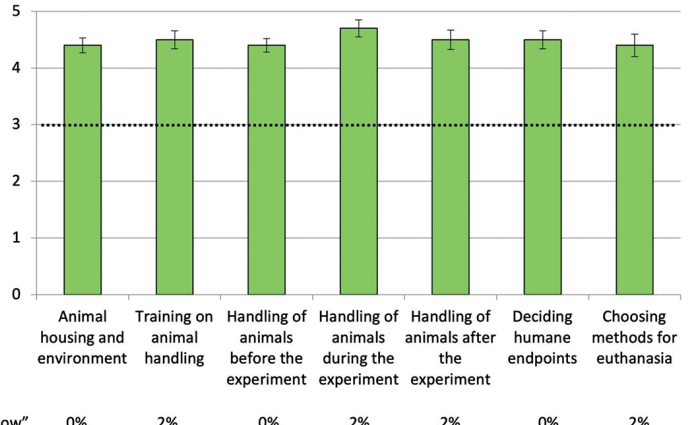

$\begin{array}{llllllll}\text { "I don't know" } & 0 \% & 2 \% & 0 \% & 2 \% & 2 \% & 0 \% & 2 \%\end{array}$

Fig. 2: Usefulness of the 3Rs during different parts of the research process according to AWB members

"The $3 R$ principles are useful in the following research situations and steps" (QI.4). Mean values and SEM of ranked results (1-5, where 1 = strongly disagree, 2 = disagree, $3=$ neither disagree/agree, 4 = agree, 5 = strongly agree). $\mathrm{N}=39-44$ (Fig. 2A), $\mathrm{N}=43-44$ (Fig. 2B). Respondents answering "I don't know" were excluded from the calculation and are presented as a percentage.

The usefulness of the 3Rs during different stages of the research process was reflected by the respondents' answers (QI.4). The respondents neither agreed nor disagreed that the $3 \mathrm{Rs}$ are useful at the beginning of the process, when the research question and hypothesis are defined, or at the final stage, when the research results are reported and published (Fig. 2A). During the stages in between, such as when optimizing study design, choosing appropriate experimental methods, planning of the experimental procedures (Fig. 2A), as well as handling animals and considering animal housing and environment, deciding humane endpoints and choosing euthanasia methods (Fig. 2B), they agreed or strongly agreed that the 3 Rs are applicable. Interestingly, $84-89 \%$ of the respondents agreed or strongly agreed that the $3 \mathrm{Rs}$ are applicable in procedures involving animals per se, i.e., housing and environment, training on handling, and when 


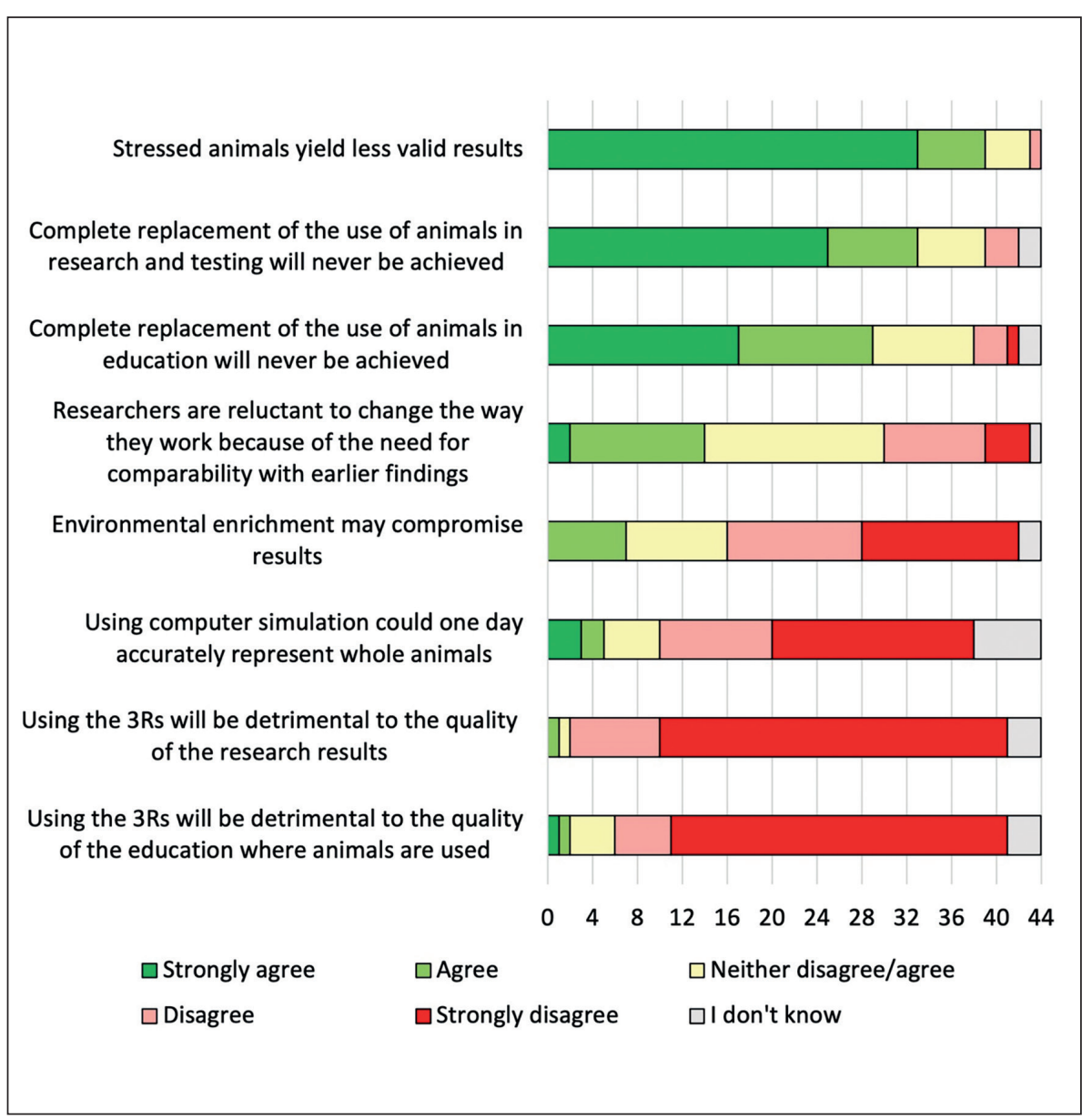

Fig. 3: AWB members' attitude towards replacement, reduction and refinement regarding scientific quality, costs and innovation rate "To what extent do you agree with the following statements?" (QI.5). Proportion of respondents answering strongly agree, agree, neither disagree/ agree, disagree, strongly disagree, I don't know. $\mathrm{N}=44$ (x-axis).

handling the animals before, during and after the experiment (Fig. 2B). In fact, none or only one of the respondents disagreed or strongly disagreed that the $3 \mathrm{Rs}$ are applicable to the handling and housing that relate to refinement. In line with that, in response to general questions (QI.5), the majority of the respondents agreed or strongly agreed that stressed animals yield less valid results and disagreed/strongly disagreed that environmental enrichment may compromise results (Fig. 3). Furthermore, in general, the respondents disagreed or strongly disagreed that the 3 Rs will be detrimental to research quality and in education where animals are used (Fig. 3). Regarding replacement, a majority of the respondents agreed or strongly agreed that the use of animals in research and education will never be completely replaced by alternative methods, and disagreed/strongly disagreed that computer simulation will represent whole animals accurately one day (Fig. 3).

\subsection{Implementation of the 3Rs}

Refinement was the $3 \mathrm{R}$ aspect considered most pertinent in research and education at the respondents' universities (36 and $23 \%$, respectively), followed by reduction (11 and $9 \%$ ), whereas none of the respondents pointed out replacement as the single most relevant aspect within research, and only $5 \%$ in educa- tion (QII.1-2). However, half of the respondents considered all three Rs equally important in both research and education (48 and $50 \%$, respectively).

The anticipated effects of the implementation of the 3Rs varied (QII.3-5). Most respondents agreed or strongly agreed that refinement results in increased research quality, whereas the answers regarding reduction and replacement were more widely distributed, from strongly agree to strongly disagree (Fig. 4). Few of the respondents agreed or strongly agreed that the 3Rs will slow down innovation (Fig. 4). Less than one third of the respondents agreed or strongly agreed that the $3 \mathrm{Rs}$ result in increased research costs (Fig. 4).

When asked what activities would enable the 3Rs at the university, $59 \%$ of the respondents answered that no activities would enable replacement because researchers look at entire animal systems, whereas increased funding to develop alternatives and help with identifying replacement techniques were suggested by 36 and 32\%, respectively (QII.8). The most important activities to reduce the number of animals used at the university were deemed to be increased sharing of data or collaboration between research groups $(63 \%)$ and greater availability of funding for 3R-related research (59\%) (QII.9). Several respondents suggested different activities that would benefit refinement: de- 


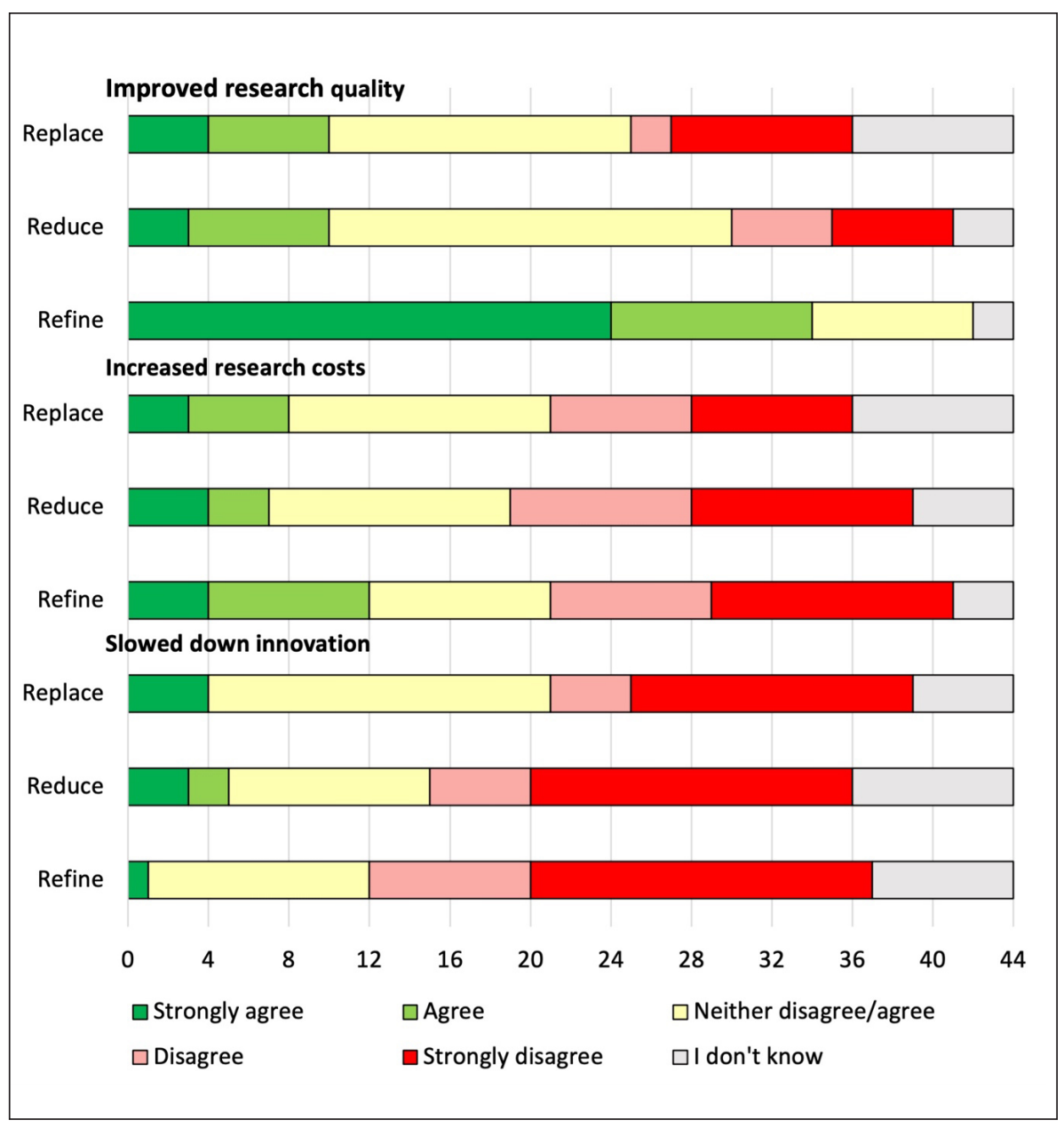

Fig. 4: AWB members' attitude towards implementation of 3R strategies at their university "To what extent do you agree with the following statements? Implementation of Replacement (QII.3), Reduction (QII.4), Refinement (QIII.5) strategies at your university results in:" Proportion of respondents answering strongly agree, agree, neither disagree/agree, disagree, strongly disagree, I don't know. $\mathrm{N}=44$ (x-axis).

velopment of best practices for housing and enrichment (73\%), greater willingness among researchers to change their research methods, increased training of animal handling, increased funding for development of refinement methods, as well as increased knowledge of ethology (59-64\%) (QII.10). 16\% and 32\% of the respondents suggested that requirements from scientific journals would benefit replacement and refinement, respectively. $18 \%$ believed that the number of animals used would decrease if scientific journals were more willing to accept non-animal methods. A minority of the respondents $(9-20 \%)$ believed that legislative or regulatory changes would benefit the $3 \mathrm{R}$ principles at the university. Regarding all 3Rs, $52 \%$ of the respondents agreed or strongly agreed that lack of financial support is an obstacle for the implementation of the 3 Rs at the university (QII.6). In contrast, the majority of the respondents disagreed or strongly disagreed that legal demands are an obstacle (57\%).

\subsection{Implementation of the EU Directive 2010/63}

This section included questions related to the designated tasks of the AWBs according to Directive 2010/63 as described in the Introduction. The AWBs stated that they do not usually give advice on the 3 Rs to staff working with animals at the universities, and most seldom on replacement (QIII.1-3) (Fig. 5). The number of respondents that claimed that they did not know was very high (32-43\%). The answers to the question asking whether the AWB keeps the staff informed of technical and scientific developments within the 3 Rs were scattered; from strongly agree to strongly disagree (QIII.4). A large proportion of respondents did not know (20\%). A smaller proportion of the respondents answered that the AWB follows the development and outcome of projects and identifies and advises on elements that further contribute to the 3R strategies; for replacement $9 \%$, for reduction $14 \%$ and for refinement $20 \%$ agreed or strongly agreed, and $20 \%$ did not know (QIII.5). 41\% percent of the respondents were positive, $25 \%$ negative and $34 \%$ did not know when asked if keeping the AWB journals open for other research groups would increase the use of the 3Rs (QIII.6).

$86 \%$ of the respondents stated that the AWBs receive input on animal welfare from designated veterinarians with expertise in laboratory animal medicine, while $25 \%$ mentioned that input is received from ethologists (QIII.7). The task of advising on rehoming animals is rarely performed (18\%), and $18 \%$ did not know if it is done (QIII.8). The answers to this question were quite contradictory, because some respondents in the AWBs at 


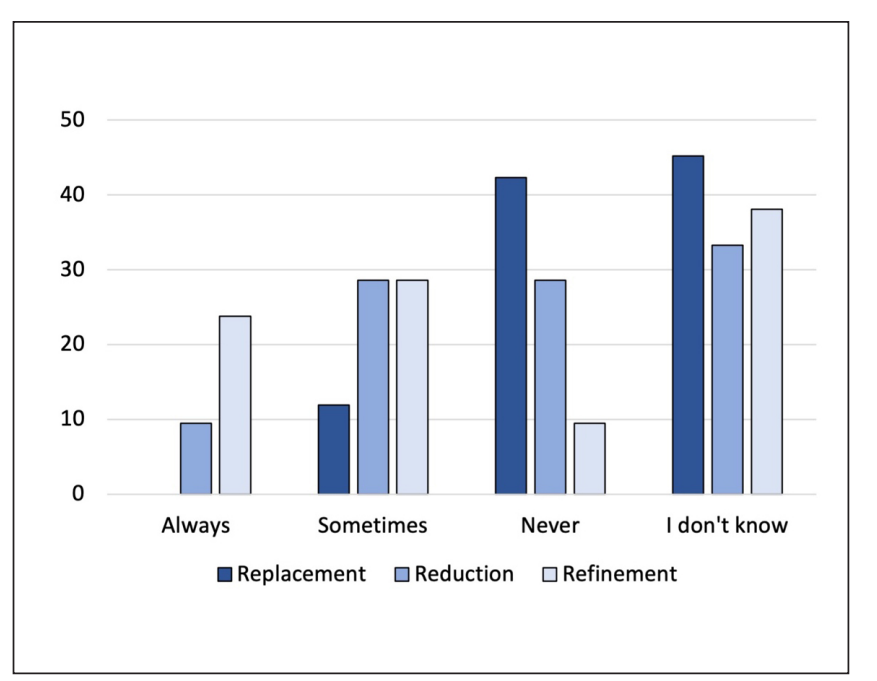

Fig. 5: Proportion of AWB members advising staff in research projects at Swedish universities on $3 \mathrm{R}$ methods/strategies "How often does your Animal Welfare Body (AWB) advise the staff on in vitro/in silico methods as Replacement for animal use in research?" (QIII.1, dark blue bars). "Does your AWB advise the staff on strategies for Reduction of animal use in research (e.g., method developments, coordination with other research groups)?" (QIII.2, medium blue bars). "Does your AWB advise the staff on methods for Refinement (e.g., environmental enrichment, improved handling and experimental techniques)?" (QIII.3, light blue bars). Always: In 10 out of 10 projects, Sometimes: In one to nine out of 10 projects (pooled answers: in five to nine out of 10 projects, in two to five out of 10 projects, in one out of 10 projects), Never: in zero out of ten projects. Responses (multiple choice, where one answer is most appropriate) presented as percentage. $\mathrm{N}=44$.

three universities stated that they give advice on rehoming, and some stated that they do not rehome animals.

\subsection{Key factors for successful implementation of the $3 R$ s at the university}

80 and $86 \%$ of respondents agreed or strongly agreed that $3 R$ awareness, education and training, and collaboration are important key factors for successful 3R development at the university. The proportions for organizational structure, management, and research and validation were 72 and $77 \%$ (QIV.1). The AWB members were then asked what would increase the awareness of the 3Rs at the university (QIV.2). The respondents agreed or strongly agreed that the university should implement the following: a $3 \mathrm{R}$ policy at the university $(59 \%)$, a budget for $3 \mathrm{R}$ implementation (68\%), the management should expect the staff to have high standards with respect to animal welfare $(72 \%)$ and encourage the staff to test $3 \mathrm{R}$ ideas $(66 \%)$, there should be mandatory $3 \mathrm{R}$ education for staff in addition to legislative requirements $(68 \%)$ and $3 \mathrm{R}$ seminars held on a regular basis at the university $(57 \%)$, and annual $3 \mathrm{R}$ goals set up by the management $(52 \%)$ to increase 3R awareness (QIV.2). A majority of the respondents agreed or strongly agreed on the importance of $3 \mathrm{R}$ awareness within the management $(80 \%)$, among researchers and animal technicians $(86 \%)$, and the staff in general $(72 \%)$ for successful implementation of the 3Rs (QIV.3).

\subsection{Differences in attitude between the professional groups in the AWB}

The $3 \mathrm{R}$ attitude of the respondents was analyzed and compared between the professional groups using ordinal logistic methods and factor analyses. All variables with numeric responses were analyzed for differences using ordinal logistic methods. Out of 140 tested variables, there were only two questions that showed a significant difference between the professional groups.

The response to "Complete replacement of the use of animals in research and testing will never be achieved" (QI.5b) differed between the groups $(p=0.033, n=44)$, where the vast majority of the researchers and persons responsible for animal welfare strongly agreed or agreed (Fig. 6A). A smaller proportion of veterinarians agreed or strongly agreed and one veterinarian disagreed. A scattered response was seen among animal technicians, which both agreed and disagreed.

Differences between the groups were also observed when asking if $3 \mathrm{R}$ research innovation, validation and implementation are important key factors for successful 3R work at their university $(\mathrm{QIV} .1 \mathrm{c})(\mathrm{p}=0.046, \mathrm{n}=44)$. Only three of nine researchers agreed, while the majority of persons responsible for animal welfare and veterinarians strongly agreed (Fig. 6B). This pattern, where the responses from the group of researchers seemed to differ from the other groups, was seen throughout the survey.

To further study this pattern, we performed factor analyses on 75 sub-questions (QI.4-5, QII.3-5, QIII.4-5, QIV.4-5). The analysis suggested that these questions can be summarized into three factors, where the highest loading above the absolute value (i.e., irrespective of its sign) of 0.3 was included in factors 1,2 and 3 , respectively. Four variables were excluded because they were less than the absolute value of 0.3 , or due to less than 0.01 difference between factors. Together these factors explained 55\% of the variation between the groups (Appendix $2^{1}$ ). The pattern was rather clear: 34 of 36 sub-questions in the pre-determined refinement category were included in Factor 1 and 2 (19 out of 34 questions in Factor 1, and 15 out of 17 questions in Factor 2). Only two refinement questions were included in Factor 3, where the other 18 sub-questions regarded replacement, reduction and general 3Rs. Negative statements in the questions resulted in negative coefficients, and positive statements resulted in positive coefficients, as anticipated.

Data were analyzed to approximate each factor with the sum of the variables that were included in it, including positive and negative variables. Differences between professional groups in the AWBs were tested for each factor: Factor 1: $\mathrm{p}=0.018$; Factor 2: $\mathrm{p}=0.814$; Factor $3: \mathrm{p}=0.341$. Interestingly, whereas differences between professional groups were noted for Factor 1, pairwise comparisons showed an evident difference between researchers and veterinarians (adjusted $\mathrm{p}=0.031$ ) (Tab. 1). This result indicates that differences between the two professional groups in the AWBs were related to questions about all three Rs, but predominately refinement (the majority of the questions in Factor 1 were 
A

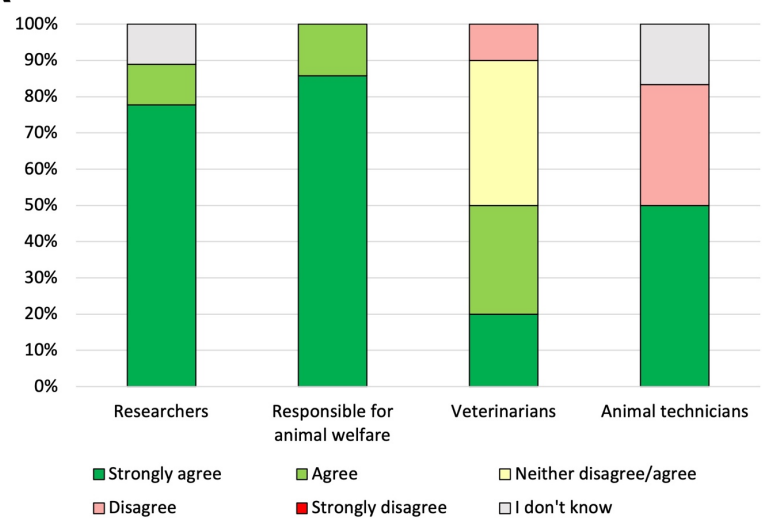

B

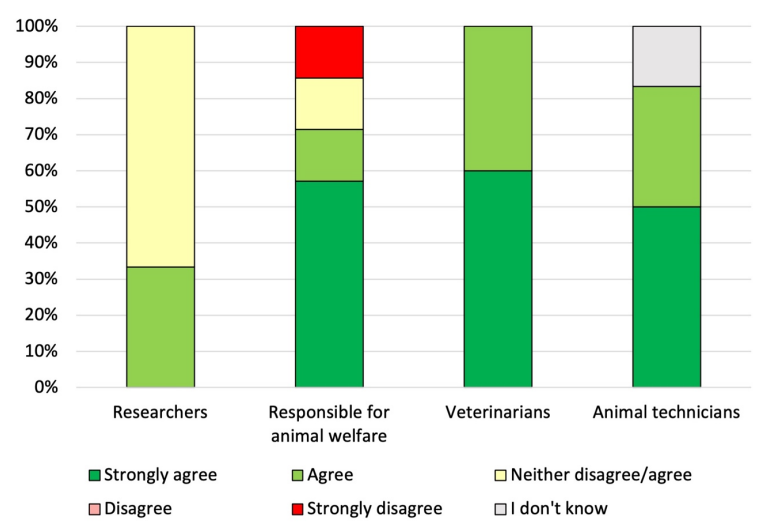

Fig. 6: Attitudes towards the 3Rs among different professional groups represented in the AWBs

(A) "Complete Replacement of the use of animals in research and testing will never be achieved" (QI.5b). Proportion (percentage of individuals in each professional group) of all respondents answering strongly agree, agree, neither disagree/agree, disagree, strongly disagree, I don't know. $N=39$ (Researchers = 9; Responsible for animal welfare = 14; Veterinarians = 10; Animal technicians = 6). (B) "The following key factors are important for successful $3 R$ work at your university: $3 R$ research innovation, validation and implementation" (QIV.1c). Proportion (percentage of individuals in each professional group) of all respondents answering strongly agree, agree, neither disagree/agree, disagree, strongly disagree, I don't know. N = 39 (Researchers = 9; Responsible for animal welfare $=14$; Veterinarians $=10 ;$ Animal technicians $=6$ ).

Tab. 1: Differences between professional groups regarding their attitudes towards the 3Rs

\begin{tabular}{|c|c|c|c|c|c|}
\hline Professional role & Professional group & $\begin{array}{l}\text { Estimates of least- } \\
\text { squares means }\end{array}$ & Standard error & t-value & Adjusted $p$ \\
\hline Researcher & $\begin{array}{l}\text { Responsible for } \\
\text { animal welfare }\end{array}$ & -12.7063 & 7.6443 & -1.66 & 0.564 \\
\hline Researcher & Veterinarian & -26.2778 & 8.2208 & -3.20 & $0.0310^{*}$ \\
\hline Researcher & Animal technician & -20.9444 & 9.4300 & -2.22 & 0.252 \\
\hline $\begin{array}{l}\text { Responsible for } \\
\text { animal welfare }\end{array}$ & Veterinarian & -13.5714 & 7.4080 & -1.83 & 0.458 \\
\hline $\begin{array}{l}\text { Responsible for } \\
\text { animal welfare }\end{array}$ & Animal technician & -8.2381 & 8.7304 & -0.94 & 0.932 \\
\hline Veterinarian & Animal technician & 5.3333 & 9.2394 & 0.58 & 0.992 \\
\hline
\end{tabular}

Factor 1: Differences between professional groups based on estimates of least-squares means after adjustment with Tukey-Kramer (DF: 38). 34 questions with the highest loading above the absolute value (i.e., irrespective of its sign) of $0.3 ;{ }^{*}$, adjusted $p<0.05$.

refinement-related while the remaining questions were split between replacement, reduction and general 3R questions, see Appendix $2^{1}$ ). Veterinarians were most positive towards the $3 \mathrm{Rs}$ according to the higher least-squares means including both positive and negative responses, and researchers were most negative. Overall, our results indicate that researchers in the AWBs have a more negative and less positive attitude towards the $3 \mathrm{Rs}$.

\section{Discussion}

Our survey provided a good picture of the understanding and implementation of the 3Rs in AWBs at all eight Swedish universities that use animals in research. We identified similarities and differences in knowledge and attitude between replacement, reduction and refinement and between the professional groups rep- 
resented in the AWBs. The implementation of Directive 2010/63 was found to be inadequate in the AWBs in our survey three years after it took effect.

\subsection{AWBs did not fully implement Directive 2010/63}

We found that the AWBs lack understanding of how to work in accordance with Directive 2010/63. Tasks of implementing 3R-related activities emphasized in the Directive were often not carried out in the AWBs or were unknown by a large proportion of the AWB members. Remarkably, more than one third of the AWB members did not know if they advise staff at the university in their research projects on methods or strategies for replacement, reduction and refinement. The European Commission has published guidance and advice for AWBs on how to work according to the Directive (EU, 2014), but our data indicates a need for more practical and regulatory guidance and support to the AWBs on national and regional levels.

In a report on the implementation of the Directive in EU member states, in general, member states, users of animals and stakeholders viewed the introduction of the AWBs positively (EU, 2017). Although more than half of the users in the report stated that there are no obstacles in delivering the tasks of the AWB, difficulties were identified, in particular concerning training, resources and insufficient authority. Despite half of the member states having changed their rehoming policies, only a limited number of animals are rehomed. In fact, there is little quantitative data on numbers, and there is no legal obligation for reporting numbers of rehomed animals (EU, 2017). This lack of organization and follow-up was observed also in our study.

According to the EU report, the introduction of AWBs increased the awareness of refinement within establishments, while replacement did not get much attention in the report (EU, 2017). This is in spite of the fact that the Directive states that full replacement of animal use for scientific and educational purposes is the longterm objective and ultimate goal (EU, 2010). In our survey, the lack of replacement activities is most pronounced when it comes to advising staff at the university on methods or strategies for their research projects. In fact, a large majority of the respondents answered that they either do not know or that advice on replacement is never given. In a previous survey, Dutch animal welfare officers were least frequently advising on replacement to researchers and most frequently on refinement (van Luijk et al., 2013). It may be easier to gather and share animal welfare and handling guidelines and standards than to gather and give advice on replacement methods for a specific research project. The required competence within the AWBs is more directed towards in vivo research rather than technical and scientific competence in replacement.

Few AWB members in our study followed the development and outcome of projects further contributing to replacement, reduction and refinement according to the EU Directive. This may have a significant impact on the use of the 3 Rs at the universities, since follow-up and feedback are key factors for successful implementation of the 3Rs within an organization (Törnqvist et al., 2014). However, according to the evaluation of Directive 2010/63, the role of AWBs needs to be clarified and not confused with project evaluation in some member states (EU, 2017). This confusion also has been evident in Sweden since 2018, when AWBs were assigned to make decisions about minor changes in ethical permit decisions issued by Swedish ethical committees (Swedish Board of Agriculture, 2019). According to member states and users in the EU, the AWBs should have oversight of the 3Rs within establishments, and the AWBs are expected to advise projects within their establishments of relevant new developments, e.g., through individual communication, newsletters and seminars. Dissemination strategies have, however, still not been developed by all AWBs (EU, 2014, 2017).

The scope of Directive 2010/63 was expanded to include animal use in education (EU, 2010; Hartung, 2010). A majority of Swedish AWB members did not think that animal use will be replaced in education. Nøhr et al. (2016) reported that a large majority of Danish researchers think the use of experimental animals for teaching at universities is acceptable. Most of the researchers participating in laboratory animal science (LAS) courses in Portugal approved of the use of animals at university level (biology, medicine and veterinary medicine), but a minority believed it necessary at high school level (Franco and Olsson, 2014). Several AEC members at Canadian universities supported the use of alternatives in teaching contrary to research (Schuppli and Fraser, 2005). Still, research animal users and stakeholders in the EU member states believed that there is significant scope for replacing animals used in education, because many alternatives are available but not well known (EU, 2017). Although undergraduate students may find the use of live animals in teaching ethically acceptable and better than the alternatives (Hunt and Macaskill, 2017), other students, e.g., in veterinary programs, favor alternative teaching practices that do not make use of animals (Silva et al., 2007; Whittaker and Anderson, 2013). In some European countries, e.g., Italy, there is an obligation to inform university students of their right to exercise their conscientious objection to educational activities involving animals (Baldelli et al., 2017).

\subsection{The knowledge of the 3Rs was generally good in Swedish AWBs}

The overall knowledge of the definitions of the 3Rs (Executive Committee of the Congress, 2009) was good among the respondents, and most often similar to findings in surveys of British researchers and animal care staff (NC3Rs, 2008) and of Danish researchers (Nøhr et al., 2016). Houde et al. (2009) also found a good overall understanding of the $3 \mathrm{Rs}$ in interviews with Canadian IACUC members, which the authors did not expect since the 3Rs were not particularly mentioned when ethical reviews were performed. Franco and Olsson (2014) reported in their survey of researchers about to participate in a LAS course that more than half of the respondents were unaware of the 3 Rs and only one of five participants could correctly name the principles. However, one year after the course, almost all respondents could still properly name the $3 \mathrm{R}$ principles.

Still, there is room for improvement. Several respondents in our survey confused reduction with refinement; fewer animals used and better data obtained as a result of improved experiments is not refinement, and reducing pain and suffering does not fall under reduction. Similar results were found by NC3Rs (2008) 
and Nøhr et al. (2016). Interviews of Canadian AEC members revealed inconsistent interpretation of the $3 \mathrm{Rs}$, but still basic understanding of replacement and reduction, while refinement appeared to be the least understood (Schuppli and Fraser, 2005). For example, researchers still sometimes incorrectly think that refinement is about the quality of scientific technique or experimental design (Hubrecht, 2014).

Like in the British and Danish studies, a small proportion of the AWB members answered that replacing vertebrates with invertebrates is not within the scope of replacement, even though invertebrates per definition are not defined as research animals in Directive 2010/63. The definition of replacing is a philosophical question about life and may vary in time and context. In fact, Russell and Burch (1959) divided replacement into absolute replacement, i.e., no animals are used (in vitro or in silico studies or in vivo studies on organisms not thought to be sentient, e.g., bacteria nematodes, amoeba, nematodes and fish larvae) and relative replacement, i.e., animals are used but without distress, e.g., non-recovery experiments under terminal anesthesia or animal tissue cultures (Hubrecht, 2014). In wildlife research, the use of species other than protected species (Lane and McDonald, 2010) or keystone species, which have a large impact on other species and ecosystems (Curzer et al., 2013), has sometimes been referred to as replacement.

\subsection{Attitudes differed between the 3Rs and between professional groups}

Interestingly, the professional groups represented in the AWB differed in their attitude towards all 3Rs. For example, our data indicate differences in opinion regarding replacement. The group of researchers did not believe that animal use in research and testing one day will be replaced with alternative methods, while most responding veterinarians seemed less negative. Purchase and Nedeva (2002) reported that a larger proportion of animal-using researchers than named veterinarians strongly believed that information from alternatives was not as valuable as from animal experiments. Knight et al. (2009) found that researchers did not believe in the existence of alternatives to animals in medical research and showed the largest support for animal use in general compared to animal welfarists and laypersons, especially for medical research. Students and potential future researchers in the Swedish toxicology MSc program at Karolinska Institutet have a strong focus on all three Rs in all courses throughout their education. In contrast to AWB members, a majority of these students believed that animal use in research can be completely replaced with alternatives (Törnqvist, unpublished data). The difference between various groups may be explained by differences in the degree of agreement with the existence of alternatives to animals, belief in humans being superior to animals, and belief in animal cognition and sentience. Indeed, the EU Directive clearly acknowledges the intrinsic value of animals (EU, 2010). In comparison, Nøhr et al. (2016) described that researchers agreed that animals have certain rights but were still in favor of using animals in experiments.

We also found in our study that the professional groups looked differently upon $3 \mathrm{R}$ research innovation, validation and imple- mentation. Researchers in the AWBs seemed less inclined to see the benefits of increased $3 \mathrm{R}$ research innovation, validation and implementation as key factors for successful 3R-work at their universities, while a larger proportion of other professional groups had a more positive attitude. Researchers may be less aware of the 3Rs because they have built their careers on animal models (Graham and Prescott, 2015).

Despite these differences between professional groups, the respondents in general most often agreed upon refinement questions in the survey, and often with a smaller proportion of "I don't know" responses compared with reduction and replacement, see e.g., Figures 2 and 4. Refinement was clearly believed to have a positive impact on research quality among the respondents in our study. With time, the understanding of animal sentience and cognition has increased, with implications for refinement. The agreed re-definition of the 3Rs in Bologna 1999 (Executive Committee of the Congress, 2009) refers to refinement strategies as methods which alleviate or minimize potential pain, suffering and distress, and which enhance animal well-being, further widening its role and relevance compared to the original definition of refinement "...to reduce to an absolute minimum the amount of distress imposed on those animals that are still used" (Russell and Burch, 1959).

Interestingly, several respondents in our study did not know the effect of replacement on research quality. Half of the responding researchers in a British survey did not believe that data from alternatives was of equal value as animal experiments (Purchase and Nedeva, 2002). This further emphasizes the need for AWBs to increase the knowledge and application of replacement. This pattern with more positive responses to refinement compared to replacement was seen for attitude and implementation, see e.g., Figures 4 and 5, but knowledge did not seem to differ between the $3 R$ s, see Figure 1.

A majority of the AWB members believed that alternative methods will never replace animal use in research. This is in accordance with results from surveys of researchers, animal care staff, and the public in other countries (NC3Rs, 2008; Fenwick et al., 2011; Leaman et al., 2014; Nøhr et al., 2016; Franco et al., 2018). The limited expectations on replacement may be explained by lack of replacement methods and technological solutions, a need for using the whole-body system, and studies where animals themselves are subject of the research (Fenwick et al., 2011; Nøhr et al., 2016). Users in EU member states indicated that studies on some aspects of biology, e.g., conscience, vigilance states, reproduction and developmental biology, continue to need in vivo experimentation, and alternative methods for these are unlikely to be available in the foreseeable future (EU, 2017). In addition, there are still legal requirements that support in vivo testing, e.g., risk assessment of chemicals (EU, 2008).

A majority of AWB members did not believe that any activities suggested in our survey, such as increased funding and support to identify replacement techniques, would benefit replacement at their university. Ditlevsen et al. (2018) found in interviews of Danish researchers that replacement method development and innovation was outside the competence and scope of Danish traditional in vivo researchers, and that time, resources, interdisci- 
plinary collaboration, and diligence would be a possible way forward to replace animal use. Attitudes to replacement may change through education. Although researchers participating in LAS courses believed in long-term dependency on animal use, the proportion of respondents answering that animal use was invaluable for every relevant research project decreased one year after learning about replacement (Franco and Olsson, 2014). Although Franco et al. (2018) found that LAS courses seemed to increase awareness of the 3 Rs, these courses had, however, no measurable effect on the low-level belief that non-animal methods can fully replace animal experimentation.

Curzer et al. (2016), pointed out that from a moral perspective the 3 Rs are hierarchical; replacement should be considered before reduction, and refinement should be considered last. In our survey, half of the AWB members stated that the 3Rs are equally relevant in research and education at their university, and a smaller number of members believed that one of the 3R principles was the single relevant one. NC3Rs (2008) and Nøhr et al. (2016) found that several respondents in their surveys ranked refinement and reduction over replacement. This was also found in a survey of researchers participating in LAS courses held in four European countries (Franco et al., 2018). The authors suggested that this ordering may conflict with the intention of the EU Directive and the interest of the public and regulators. Indeed, replacement seems to be the hardest R to fully grasp and embrace for researchers. Although several Canadian researchers said that they are using the $3 \mathrm{Rs}$ as much as possible, they found replacement difficult to implement, and less applicable than reduction and refinement (Fenwick et al., 2011). Researchers may often see animal-free methods as complementary to animal research models, rather than as complete replacement (Knight et al., 2009). Thus, traditional replacement strategies, i.e., in vitro and in silico methods, result in reduction as well as replacement (Törnqvist et al., 2014).

\subsection{Organization, management and awareness are key factors for successful 3R development}

Factors such as organizational structure and management were considered by the AWBs to be important factors for successful implementation of the 3Rs. Graham and Prescott (2015) suggested that in a well-run animal facility, investigators, their scientific peers, the attending veterinarian and animal care staff should adopt a team approach when working towards the 3Rs. Törnqvist et al. (2014) showed that organizational culture and 3R awareness are crucial for successful implementation of the $3 R s$ in the daily practices of researchers and animal care staff. In fact, Brønstad and Berg (2011) suggested that organizational culture is more important than legal demands to achieve $3 \mathrm{R}$ improvements, especially if legislation is perceived as ineffective. High expectations from the management in organizations stimulate staff to act on and test $3 \mathrm{R}$ ideas. In addition, tools to facilitate the validation process from innovation to implementation should be provided, as suggested by Törnqvist et al. (2014) and Lindsjö et al. (2016). Still, researchers believe that replacement is especially difficult to develop and validate (NC3Rs, 2008; Fenwick et al., 2011; Nøhr et al., 2016).
AWB members identified 3R awareness as another important factor for successful implementation of the 3Rs, and several activities to increase the awareness. Based on our findings in this study, it is clear that increased $3 \mathrm{R}$ awareness is needed for all professional roles in animal use for scientific and educational purposes. A strong culture of care within the organization will increase the $3 \mathrm{R}$ awareness. 3R visions and goals, education and training, and recognition of good $3 \mathrm{R}$ examples may further increase awareness. Nevertheless, application of the 3Rs when planning and carrying out experiments should be part of a research organization's goal (Brønstad and Berg, 2011; Smith et al., 2018). To increase 3R awareness, 3R methods should be shared internally, e.g., through individual communications, newsletters and 3R seminars (EU, 2017) and published to enable sharing of information and collaborations among users and different stakeholders (Brønstad and Berg, 2011). In addition, several respondents in this survey believed that keeping AWB journals open for other research groups would increase 3R use. Brønstad and Berg (2011) encouraged researchers to include the $3 \mathrm{Rs}$ as key words in publications, even when these principles were not the aims of the research.

AWBs play a crucial role in promoting $3 \mathrm{R}$ awareness because they should ensure continuous application of the $3 \mathrm{Rs}$ in care and use of animals within the establishment (EU, 2017). Users in member states reported that the AWB has had a positive impact in improving the culture of care, e.g., by increasing staff and improving staff quality by better training, improving communications between them, and improving 3R teamwork, thus optimizing animal welfare and ensuring robust scientific output (EU, 2017). If the AWBs are properly resourced and their decisions are supported by establishment management, they can deliver the requirements and aspirations of Directive 2010/63 (EU, 2017).

\section{Conclusions and recommendations}

The AWBs at Swedish universities were at the time of the survey often not fully familiar with the requirements of Directive 2010/63, and tasks required of them were rarely or incompletely performed. Several key areas were considered to be important for implementation of the 3 Rs at their universities. AWBs play an important role to ensure and increase 3R awareness and implementation at Swedish universities, and there is much untapped potential to achieve this.

The understanding of the 3 Rs was generally good, and the overall attitude towards the 3Rs was positive in AWBs at Swedish universities. AWB members did not believe that the 3 Rs slow down innovation or result in increased costs, and refinement was considered beneficial for research quality. The respondents were positive towards refinement questions regarding general attitude and implementation of the EU Directive. A majority of the AWB members believed that alternative methods will never replace animal use, i.e., that complete replacement is difficult to achieve. Researchers as a group represented in the AWBs were the least positive towards the $3 \mathrm{Rs}$, while veterinarians were the most positive. 
Based on the present study and related publications in the area, we suggest national support to AWBs regarding all required tasks according to the EU Directive. To further strengthen the AWBs at Swedish universities, local support from management and organization is essential, for example a local 3R policy, or a 3R agreement for researchers at the university to sign before performing studies, annual goals and recognition of staff promoting the development of the 3Rs (3R awards), and educational activities to strengthen 3R interest and knowledge among staff.

We suggest continued efforts to increase $3 \mathrm{R}$ awareness and interest in the research community with education and expectations of increased 3R standards at the universities as well as external whips and carrots, e.g., 3R rating and implications by research funding agencies and scientific journals. To reach the goal of the EU Directive to phase out the use of animals in research and education, special efforts should be made to increase the trust in replacement strategies among researchers, funding agencies and scientific publishers. To further enhance replacement strategies at the universities, we suggest that technical expertise outside the traditional biological sciences is included in the AWBs.

\section{References}

Baldelli, I., Massaro, A., Penco, S. et al. (2017). Conscientious objection to animal experimentation in Italian Universities. Animals (Basel) 7, 24. doi:10.3390/ani7030024

Brønstad, A. and Berg, A. G. (2011). The role of organizational culture in compliance with the principles of the 3Rs. Lab Anim (NY) 40, 22-26. doi:10.1038/laban0111-22

Curzer, H. J., Wallace, M. C., Perry, G. et al. (2013). The ethics of wildlife research: A nine R theory. ILAR J 54, 52-57. doi:10.1093/ilar/ilt012

Curzer, H. J., Perry, G., Wallace, M. C. et al. (2016). The three Rs of animal research: What they mean for the institutional animal care and use committee and why. Sci Eng Ethics 22, 549565. doi:10.1007/s11948-015-9659-8

Ditlevsen, K., Lund, T. B. and Lassen, J. (2018). Replacement $i$ forsøgsdyrsforskningen? En kvalitativ analyse af muligheder og barrierer. Frederiksberg: Institut for Fødevare- og Ressourceøkonomi, Københavns Universitet. IFRO Rapport, Nr. 270 [Article in Danish].

EU - European Union (2008). Council Regulation (EC) No 440/2008 of 30 May 2008 laying down test methods pursuant to Regulation (EC) No 1907/2006 of the European Parliament and of the Council on the Registration, Evaluation, Authorisation and Restriction of Chemicals (REACH). OJ L 142, 1-739.

EU (2010). Directive 2010/63/EU of the European Parliament and of the Council of 22 September 2010 on the protection of animals used for scientific purposes. OJ L 276, 33-79.

EU (2014). Caring for animals aiming for better science. National Competent Authorities for the implementation of Directive 2010/63/EU on the protection of animals used for scientific purposes - A working document on Animal Welfare Bodies and National Committees to fulfil the requirements under the Directive. Brussels, 9-10 October 2014.

EU (2017). Commission staff working document Accompany- ing the document Report from the commission to the European Parliament, the Council, the European economic and social committee and the Committee of the regions In accordance with Article 58 of Directive 2010/63/EU on the protection of animals used for scientific purposes. COM/2017/0631 final.

Executive Committee of the Congress (2009). The three Rs declaration of Bologna, adopted by the third world congress on alternative and animal use in the life sciences, Bologna, Italy, on 31 August 1999. Altern Lab Anim 37, 286-289. doi:10.1177/026119290903700310

Fenwick, N., Danielson, P. and Griffin, G. (2011). Survey of Canadian animal-based researchers' views on the three Rs: Replacement, reduction and refinement. PLoS One 6, 22478. doi:10.1371/journal.pone.0022478

Franco, N. H. and Olsson, I. A. (2014). Scientists and the 3Rs: Attitudes to animal use in biomedical research and the effect of mandatory training in laboratory animal science. Lab Anim 48, 50-60. doi:10.1177/0023677213498717

Franco, N. H., Sandøe, P. and Olsson, I. A. S. (2018). Researchers' attitudes to the 3Rs - An upturned hierarchy? PLoS One 13, e0200895. doi:10.1371/journal.pone.0200895

Graham, M. L. and Prescott, M. J. (2015). The multifactorial role of the 3Rs in shifting the harm-benefit analysis in animal models of disease. Eur J Pharmacol 759, 19-29. doi:10.1016/j. ejphar.2015.03.040

Hartung, T. (2010). Comparative analysis of the revised Directive 2010/63/EU for the protection of laboratory animals with its predecessor 86/609/EEC - A t ${ }^{4}$ report. ALTEX 27, 285-303. doi:10.14573/altex.2010.4.285

Houde, L., Dumas, C. and Leroux, T. (2009). Ethics: Views from IACUC members. Altern Lab Anim 37, 291-296. doi:10.1177/026119290903700311

Hubrecht, R. (2014). The Welfare of Animals Used in Research: Practice and Ethics. Ames, Iowa, USA: John Wiley \& Sons Inc.

Hunt, M. J. and Macaskill, M. C. (2017). Student responses to active learning activities with live and virtual rats in psychology teaching laboratories. Teach Psychol 44, 160-164. doi:10.1177/0098628317692632

Knight, S., Vrij, A., Bard, K. et al. (2009). Science versus human welfare? Understanding attitudes toward animal use. J Soc Issues 65, 463-483. doi:10.1111/j.1540-4560.2009.01609.x

Lane, J. M. and McDonald, R. A. (2010). Welfare and 'best pract tice' in field studies of wildlife. In R. Hubrecht and J. Kirkwood (eds.), The UFAW Handbook on the Care and Management of Laboratory and Other Research Animals (92-106). $8^{\text {th }}$ edition. Oxford, UK: Wiley-Blackwell. doi:10.1002/9781444318777

Leaman, J., Latter, J. and Clemence, M. (2014). Attitudes to animal research in 2014. A report by Ipsos MORI for the Department for Business, Innovation \& Skills. 14-012982-01. https:// bit.ly/32I2utw

Leenaars, M., Savenije, B., Nagtegaal, A. et al. (2009). Assessing the search for and implementation of the three Rs: A survey among scientists. Altern Lab Anim 37, 297-303. doi:10.1177/026119290903700312

Lindsjö, J., Fahlman, Å. and Törnqvist, E. (2016). Animal welfare from mouse to moose - Implementing the principles 
of the 3Rs in wildlife research. Jour Wildl Dis 52, S65-S77. doi: $10.7589 / 52.2$ S.S65

Ljung, P. E. and Bornestaf, C. (2018). Användning av försöksdjur i Sverige under 2016. Jordbruksverket Rapport Dnr: 5.2.17-12670/17 [Article in Swedish].

Morrison, D. F. (1976). Multivariate Statistical Methods. $2^{\text {nd }}$ edition. New York, NY, USA: McGraw-Hill.

NC3Rs - National Centre for the Replacement, Refinement and Reduction of Animals in Research (2008). Views on the 3Rs, Survey Report - 2008. London, UK.

NORECOPA - Norwegian Consensus Platform for Replacement Reduction and Refinement of Animal Experiments (2012). Harmonisation of the Care and Use of Agricultural Animals in Research. Gardermoen, 26-28 September 2012. A consensus document from the participants. https://norecopa.no/ media/6323/consensus-statement-agricultural-animals.pdf

Nøhr, R., Lund, T. B. and Lassen, J. (2016). The Danish 3R survey: Knowledge, attitudes and experiences with the 3Rs among researchers involved in animal experiments in Denmark. Fredriksberg: Department of Food and ResourceEconomics, University of Copenhagen. IFRO Report, No. 249.

Olsson, U. (1979). Maximum likelihood estimation of the polychoric correlation coefficient. Psychometrika 44, 443-460. doi:10.1007/BF02296207

Olsson, U. (2002). Generalized Linear Models: An Applied Approach. Lund, Sweden: Student-litteratur.

Purchase, I. F. H. and Nedeva, M. (2002). The impact of the introduction of the ethical review process for research using animals in the UK: Implementation of policy. Lab Anim 36, 6885. doi:10.1258/0023677021911786

Russell, W. M. S. and Burch, R. L. (1959). The Principles of Humane Experimental Technique. London, UK: Methuen \& Co. Ltd.

SAS Institute Inc. (2017). SAS/Stat User's Guide. Version 9.4. Cary, N.C., SAS Institute Inc.

Schiffelers, M. J., Blaauboer, B. J., Fentener van Vlissingen, J. M. et al. (2007). Factors stimulating or obstructing the implementation of the $3 \mathrm{Rs}$ in the regulatory process. ALTEX 24, 271-833. doi:10.14573/altex.2007.4.271

Schuppli, C. A. and Fraser, D. (2005). The interpretation and application of the three Rs by animal ethics commit- tee members. Altern Lab Anim 33, 487-500. doi:10.1177/ 026119290503300511

Silva, R. M. G., Matera, J. M. and Ribeiro, A. A. C. M. (2007). New alternative methods to teach surgical techniques for veterinary medicine students despite the absence of living animals. Is that an academic paradox? Anat Histol Embryol 36, 220-224. doi:10.1111/j.1439-0264.2007.00759.x

Smith, A. J., Clutton, R. E., Lilley, E. et al. (2018). PREPARE: Guidelines for planning animal research and testing. Lab Anim 52,135-141. doi:10.1177/0023677217724823

Swedish Board of Agriculture (2019). Swedish Regulations and General Recommendations (SJVFS 2019:9) on Research Animals, case no L150 [Article in Swedish]. https://lagen.nu/ sjvfs/2019:9

Törnqvist, E., Annas, A., Granath, B. et al. (2014). Strategic focus on $3 \mathrm{R}$ principles reveals major reductions in the use of animals in pharmaceutical toxicity testing. PLoS One 9, e101638. doi:10.1371/journal.pone. 0101638

van Luijk, J., Cuijpers, Y., van der Vaart, L. et al. (2011). Assessing the search for information on three Rs methods, and their subsequent implementation: A national survey among scientists in the Netherlands. Altern Lab Anim 39, 429-447. doi:10.1177/026119291103900505

van Luijk, J., Cuijpers, Y., van der Vaart, L. et al. (2013). Assessing the application of the 3Rs: A survey among animal welfare officers in the Netherlands. Lab Anim 47, 210-219. doi:10.1177/0023677213483724

Whittaker, A. L. and Anderson, G. I. (2013). A policy at the University of Adelaide for student objections to the use of animals in teaching. $J$ Vet Med Edu 40, 52-57. doi:10.3138/ jvme.0411.045R1

\section{Conflict of interest}

The authors have no conflicts of interest.

\section{Acknowledgements}

We thank the Swedish Association for the Protection of Animals for financial support and Jonas Förare and Ulf Norinder for technical support. 